\title{
COMPACT HERMITIAN SURFACES OF POINTWISE CONSTANT HOLOMORPHIC SECTIONAL CURVATURE
}

\author{
by KOUEI SEKIGAWA and TAKASHI KODA
}

(Received 7 April, 1994)

1. Introduction. Let $M=(M, J, g)$ be an almost Hermitian manifold and $U(M)$ the unit tangent bundle of $M$. Then the holomorphic sectional curvature $H=H(x)$ can be regarded as a differentiable function on $U(M)$. If the function $H$ is constant along each fibre, then $M$ is called a space of pointwise constant holomorphic sectional curvature. Especially, if $H$ is constant on the whole $U(M)$, then $M$ is called a space of constant holomorphic sectional curvature. An almost Hermitian manifold with an integrable almost complex structure is called a Hermitian manifold. A real 4-dimensional Hermitian manifold is called a Hermitian surface. Hermitian surfaces of pointwise constant holomorphic sectional curvature have been studied by several authors (cf. [2], [3], [5], [6] and so on).

In this paper, we shall prove the following.

TheOREm A. Let $M=(M, J, g)$ be a compact Hermitian surface of pointwise constant holomorphic sectional curvature. If the scalar curvature of $M$ is nonpositive constant, then $M$ is an Einstein Kähler surface.

THEOREM B. Let $M=(M, J, g)$ be a Hermitian surface of pointwise constant holomorphic sectional curvature satisfying the condition

$$
R(X, Y) \cdot R=0 \text { for any differentiable vector fields } X \text { and } Y .
$$

If the curvature operator is non-singular at each point of $M$, then $M$ is a weakly *-Einstein manifold.

Taking account of the solution of Yamabe's problem, the classification problem of compact self-dual (resp. anti-self-dual) Hermitian surfaces can be reduced to the one of compact self-dual (resp. anti-self-dual) Hermitian surfaces with constant scalar curvature. We may easily show that a 4-dimensional almost Hermitian manifold of pointwise constant holomorphic sectional curvature is self-dual (cf. [2]). Therefore Theorem A gives a partial solution to the classification problem of compact self-dual Hermitian surfaces and also a partial improvement to the previous result of the present authors ([3], Theorem A). In the course of the proof, we have used the following fact ([3], Proposition 2.1).

Proposition. [3] Let $M=(M, J, g)$ be a compact Einstein Hermitian surface of pointwise constant holomorphic sectional curvature. Then $M$ is a locally conformal Kähler surface and the tensor field $S$ defined by

$$
S(X, Y)=\left(\nabla_{X} \omega\right) Y-\left(\nabla_{J X} \omega\right) J Y+\frac{1}{2}(\omega(X) \omega(Y)-\omega(J X) \omega(J Y))
$$

vanishes on $M$.

However the proof of the proposition is not right (more precisely, the equality (2.19)

1991 Mathematics Subject Classification. 53C55, 53C25, 53C15.

Glasgow Math. J. 37 (1995) 343-349. 
in [3] is false in sign), which has been pointed out by T. Sato. We give a correct proof of the proposition after proving Theorem A and B.

The authors wish to express their gratitude to Professor T. Sato for his useful advice.

2. Preliminaries. Let $M=(M, J, g)$ be a $2 n$-dimensional almost Hermitian manifold with the almost Hermitian structure $(J, g)$, and $\Omega$ the Kähler form of $M$ defined by $\Omega(X, Y)=g(X, J Y), X, Y \in \mathscr{L}(M)$. We assume that $M$ is oriented by the volume form $d M=\frac{(-1)^{n}}{n !} \Omega^{n}$. We denote by $\nabla, R, \rho, \tau, \rho^{*}$ and $\tau^{*}$ the Riemannian connection, the Riemannian curvature tensor, the Ricci tensor, the scalar curvature, the $*$-Ricci tensor and the *-scalar curvature of $M$ respectively:

$$
\begin{aligned}
R(X, Y) & =\left[\nabla_{X}, \nabla_{Y}\right]-\nabla_{[X, Y]}, \\
\rho(x, y) & =\text { trace of }(z \rightarrow R(z, x) y), \\
\tau & =\text { trace of } \rho, \\
\rho^{*}(x, y) & =\frac{1}{2} \text { trace of }(z \rightarrow R(x, J y) J z), \\
\tau^{*} & =\text { trace of } \rho^{*},
\end{aligned}
$$

where $X, Y \in \mathscr{X}(M), x, y, z \in T_{p}(M), p \in M$.

An almost Hermitian manifold $M=(M, J, g)$ is called a weakly *-Einstein manifold if it satisfies $\rho^{*}=\lambda^{*} g$ for some function $\lambda^{*}$ on $M$.

Now we assume that $M$ is a Hermitian surface. Then we have

$$
d \Omega=\omega \wedge \Omega,
$$

where $\omega=\delta \Omega \circ J$. The 1-form $\omega$ is called the Lee form of $M$. The Lee form $\omega$ satisfies the following (see [7], [8]):

$$
\begin{aligned}
J^{i j} \nabla_{i} \omega_{j} & =0, \\
2 \nabla_{i} J_{j k} & =\omega_{a} J_{j}^{a} g_{i k}-\omega_{a} J_{k}{ }^{a} g_{i j}+\omega_{j} J_{k i}-\omega_{k} J_{j i}, \\
\tau-\tau^{*} & =2 \delta \omega+\|\omega\|^{2} .
\end{aligned}
$$

Let $M$ be a Hermitian surface of pointwise constant holomorphic sectional curvature $c=c(p)(p \in M)$. Then we have (see [5])

where

$$
\begin{aligned}
R_{i j k l}= & \frac{1}{4}\|\omega\|^{2} C_{i j k l}+\left(\frac{c}{4}-\frac{1}{16}\|\omega\|^{2}\right) H_{i j k l} \\
& +\frac{1}{96}\left\{g_{i k} A_{j l}-g_{i l} A_{j k}+g_{j l} A_{i k}-g_{j k} A_{i l}\right. \\
& +J_{i k} B_{j l}-J_{i l} B_{j k}+J_{j l} B_{i k}-J_{j k} B_{i l} \\
& \left.+2 J_{i j} B_{k l}+2 J_{k l} B_{i j}\right\},
\end{aligned}
$$

$$
\begin{aligned}
C_{i j k l} & =g_{i l} g_{j k}-g_{i k} g_{j l}, \\
H_{i j k l} & =g_{i l} g_{j k}-g_{i k} g_{j l}+J_{i l} J_{j k}-J_{i k} J_{j l}-2 J_{i j} J_{k l}, \\
A_{i j} & =21\left(\nabla_{i} \omega_{j}+\nabla_{j} \omega_{i}+\omega_{i} \omega_{j}\right)-3 J_{i}^{a} J_{j}^{b}\left(\nabla_{a} \omega_{b}+\nabla_{b} \omega_{a}+\omega_{a} \omega_{b}\right), \\
B_{i j} & =7\left(J_{j}^{a} \nabla_{i} \omega_{a}-J_{i}^{a} \nabla_{j} \omega_{a}\right)-\left(J_{j}^{a} \nabla_{a} \omega_{i}-J_{i}^{a} \nabla_{a} \omega_{j}\right)+3\left(J_{j}^{a} \omega_{i} \omega_{a}-J_{i}^{a} \omega_{j} \omega_{a}\right) .
\end{aligned}
$$


We put

$$
\begin{aligned}
& T_{i j}=\nabla_{i} \omega_{j}+\nabla_{j} \omega_{i}+\omega_{i} \omega_{j}-J_{i}^{a} J_{j}^{b}\left(\nabla_{a} \omega_{b}+\nabla_{b} \omega_{a}+\omega_{a} \omega_{b}\right), \\
& T_{i j}^{*}=\nabla_{i} \omega_{j}-\nabla_{j} \omega_{i}-J_{i}^{a} J_{j}^{b}\left(\nabla_{a} \omega_{b}-\nabla_{b} \omega_{a}\right)
\end{aligned}
$$

Then we have

$$
\begin{gathered}
\rho=\frac{\tau}{4} g-\frac{1}{4} T, \\
\rho^{*}=\frac{\tau^{*}}{4} g+\frac{1}{4} T^{*} .
\end{gathered}
$$

We may easily get (see [5])

$$
c=\frac{\tau+3 \tau^{*}}{24}
$$

We have the following integral formula (see [5]).

$$
\int_{M}\|T\|^{2} d M=\int_{M}\left(4\|d \omega\|^{2}+2\left(\tau-\tau^{*}\right)^{2}-4 \tau^{*}\|\omega\|^{2}\right) d M .
$$

Proposition 2.1. [5] Let $M$ be a compact Hermitian surface of pointwise constant holomorphic sectional curvature $c$. Then the Euler class of $M$ is given by

$$
\chi(M)=\frac{1}{32 \pi^{2}} \int_{M}\left\{12 c^{2}-\frac{1}{16}\left(\tau-\tau^{*}\right)^{2}+\frac{1}{2} \tau^{*}\|\omega\|^{2}\right\} d M .
$$

Proposition 2.2. [5] Let $M$ be a compact Hermitian surface of pointwise constant holomorphic sectional curvature. Then the square of the first Chern class of $M$ is given by

$$
c_{1}(M)^{2}=\frac{1}{32 \pi^{2}} \int_{M}\left\{\left(\tau^{*}\right)^{2}+\tau^{*}\|\omega\|^{2}+\|d \omega\|^{2}\right\} d M .
$$

TheOREM 2.3. [4] Let $M=(M, J)$ be a compact connected complex surface. Then we have

$$
c_{1}(M)^{2} \leqq \max \left\{2 c_{2}(M), 3 c_{2}(M)\right\}
$$

3. Proof of Theorem A. In this section, we shall prove Theorem A. Before proceeding to the proof, we recall the following fact.

THEOREM 3.1. [5] Let $M=(M, J, g)$ be a compact Hermitian surface of constant nonpositive holomorphic sectional curvature. Then $M$ is a Kähler surface.

We assume that $M=(M, J, g)$ is a compact Hermitian surface of pointwise constant holomorphic sectional curvature $c=c(p), p \in M$. 
First we assume that $c_{2}(M)(=\chi(M))<0$. Then Miyaoka's inequality (2.8) implies $c_{1}(M)^{2} \leqq 2 c_{2}(M)$. Then by $(2.4),(2.6)$ and $(2.7)$, we have

$$
\begin{aligned}
0 & \leqq \int_{M}\left\{\frac{1}{24}\left(\tau+3 \tau^{*}\right)^{2}-\frac{1}{8}\left(\tau-\tau^{*}\right)^{2}+\tau^{*}\|\omega\|^{2}-\left(\tau^{*}\right)^{2}-\tau^{*}\|\omega\|^{2}-\|d \omega\|^{2}\right\} d M \\
& =\int_{M}\left\{\frac{1}{24}\left(-2 \tau^{2}+12 \tau \tau^{*}-18\left(\tau^{*}\right)^{2}\right)-\|d \omega\|^{2}\right\} d M \\
& =\int_{M}\left\{-\frac{1}{12}\left(\tau-3 \tau^{*}\right)^{2}-\|d \omega\|^{2}\right\} d M \leqq 0 .
\end{aligned}
$$

Thus we have

$$
\tau=3 \tau^{*} \text { and } \quad d \omega=0
$$

In this case, by the assumption that $M$ has nonpositive constant scalar curvature $\tau, c$ is nonpositive constant on $M$. By Theorem 3.1, $M$ is a Kähler surface. And then we have $\tau=\tau^{*}=c=0$. This contradicts $\chi(M)<0$.

Hence it follows that $c_{2}(M)(=\chi(M)) \geqq 0$. Then Miyaoka's inequality implies

$$
c_{1}(M)^{2} \leqq 3 c_{2}(M) \text {. }
$$

Then from (2.4), (2.6) and (2.7), we have

$$
\begin{aligned}
0 \leqq & \int_{M}\left\{\frac{1}{16}\left(\tau+3 \tau^{*}\right)^{2}-\frac{3}{16}\left(\tau-\tau^{*}\right)^{2}+\frac{3}{2} \tau^{*}\|\omega\|^{2}-\left(\tau^{*}\right)^{2}-\tau^{*}\|\omega\|^{2}\right. \\
& \left.-\frac{1}{4}\|T\|^{2}+\frac{1}{2}\left(\tau-\tau^{*}\right)^{2}-\tau^{*}\|\omega\|^{2}\right\} d M .
\end{aligned}
$$

From (3.2) and (2.5) we have

$$
\begin{aligned}
\int_{M}\left\{\frac{1}{16}\left(\tau+3 \tau^{*}\right)^{2}-\left(\tau^{*}\right)^{2}+\frac{1}{16}\right. & \left.\left(\tau-\tau^{*}\right)^{2}\right\} d M \\
& \geqq \int_{M}\left\{\frac{1}{2} \tau^{*}\|\omega\|^{2}-\frac{1}{4}\left(\tau-\tau^{*}\right)^{2}+\frac{1}{8}\|T\|^{2}\right\} d M+\frac{1}{8} \int_{M}\|T\|^{2} d M \\
& =\frac{1}{2} \int_{M}\|d \omega\|^{2} d M+\frac{1}{8} \int_{M}\|T\|^{2} d M \geqq 0
\end{aligned}
$$

The left hand side of the above inequality reduces to

$$
\begin{aligned}
\int_{M} & \left(\left(\frac{1}{4}\left(\tau+3 \tau^{*}\right)-\tau^{*}\right)\left(\frac{1}{4}\left(\tau+3 \tau^{*}\right)+\tau^{*}\right)+\frac{1}{16}\left(\tau-\tau^{*}\right)^{2}\right) d M \\
& =\frac{1}{16} \int_{M}\left(\left(\tau-\tau^{*}\right)\left(\tau+7 \tau^{*}\right)+\left(\tau-\tau^{*}\right)^{2}\right) d M \\
& =-\frac{3}{8} \int_{M}\left(\tau-\tau^{*}\right)^{2} d M+\frac{\tau}{2} \int_{M}\left(\tau-\tau^{*}\right) d M \\
& =-\frac{3}{8} \int_{M}\left(\tau-\tau^{*}\right)^{2} d M+\frac{\tau}{2} \int_{M}\|\omega\|^{2} d M \leqq 0
\end{aligned}
$$


Thus by (3.3) and (3.4), we have finally $d \omega=0, T=0$ and hence $S=0$, where $S$ is the tensor field defined by

$$
S(X, Y)=\left(\nabla_{X} \omega\right) Y-\left(\nabla_{J X} \omega\right) J Y+\frac{1}{2}(\omega(X) \omega(Y)-\omega(J X) \omega(J Y))
$$

Thus, from (2.3), we see that $M$ is an Einstein locally conformal Kähler surface and the tensor field $S$ vanishes on $M$. In particular, Proposition 1.2 of [3] is valid in the case where the Einstein constant is nonpositive. Thus by the argument after Proposition 2.1 of [3], we may conclude that $M$ is Kähler surface.

This completes the proof of Theorem A.

4. Proof of Theorem B. In this section, we shall prove Theorem B. The condition (1.1) implies

$$
R_{i j a}{ }^{t} R_{t b c d}+R_{i j b}{ }^{t} R_{a t c d}+R_{i j c}{ }^{\prime} R_{a b t d}+R_{i j d}{ }^{t} R_{a b c t}=0
$$

Now by (2.3) we have

$$
\begin{aligned}
J^{i a} J^{j c} R_{i j a}{ }^{t} R_{t b c d} & =\frac{1}{2} J^{i a} J^{j c}\left(R_{i j a}{ }^{t}-R_{a j i}{ }^{t}\right) R_{t b c d} \\
& =-\frac{1}{2} J^{i a} J^{j c} R_{a i j}{ }^{t} R_{t b c d} \\
& =-\rho^{* t c} R_{t b c d} \\
& =\frac{\tau^{*}}{4} \rho_{b d}-\frac{1}{4} T^{* t c} R_{t b c d} \\
& =\frac{\tau^{*}}{4} \rho_{b d}-\frac{1}{8} T^{* t c}\left(R_{t b c d}-R_{c b t d}\right) \\
& =\frac{\tau^{*}}{4} \rho_{b d}-\frac{1}{8} T^{* t c} R_{t c b d}, \\
J^{i a} J^{j c} R_{i j c}{ }^{t} R_{a b t d} & =\frac{1}{2} J^{i a} J^{j c}\left(R_{i j c}{ }^{t}-R_{i c j}{ }^{\prime}\right) R_{a b t d} \\
& =-\frac{1}{2} J^{i a j j c} R_{j c i}{ }^{t} R_{a b t d} \\
& =\rho^{* t a} R_{a b t d} \\
& =-\frac{\tau^{*}}{4} \rho_{b d}-\frac{1}{8} T^{* t a} R_{t a b d},
\end{aligned}
$$




$$
\begin{aligned}
J^{i a}{ }^{j c} R_{i j b}{ }^{t} R_{a t c d} & =\frac{1}{2} J^{i a} J^{j c} R_{i j b}{ }^{t}\left(R_{a t c d}-R_{c t a d}\right) \\
& =-\frac{1}{2} J^{i a j j c} R_{i j b}{ }^{t} R_{c a t d} \\
& =-\frac{1}{2} J^{i a} J^{j c} R_{i j b}{ }^{t} R_{a c d t}, \\
J^{i a}{ }^{j c} R_{i j d}{ }^{t} R_{a b c t} & =\frac{1}{2} J^{i a} J^{j c} R_{i j d}{ }^{t}\left(R_{a b c t}-R_{c b a t}\right) \\
& =-\frac{1}{2} J^{i a} J^{j c} R_{i j d}{ }^{t} R_{c a b t} \\
& =\frac{1}{2} J^{a i j} J^{c j} R_{i j d}{ }^{t} R_{a c b t} \\
& =\frac{1}{2} J^{i a} J^{i c} R_{a c d}{ }^{t} R_{i j b t .}
\end{aligned}
$$

Thus, transvecting (4.1) with $J^{i a} J^{j c}$ and taking account of (4.2)-(4.5), we have

$$
R_{a b c d} T^{* a b}=0 .
$$

Since the curvature operator is non-singular at each point of $M,(4.6)$ implies $T^{*}=0$ on $M$. Hence by $(2.3)$ we see that $M$ is a weakly $*$-Einstein manifold.

This completes the proof of Theorem B.

Finally we shall prove Proposition 2.1 of [3]. We assume that $M$ is a compact Einstein Hermitian surface of pointwise constant holomorphic sectional curvature $c=c(p)(p \in$ $M)$. Taking account of the proof of Theorem A in Section 3, it suffices to consider the case where $\tau>0$. N. Hitchin proved the following.

THEOREM 4.1. [1] Let $M=(M, g)$ be a 4-dimensional half-conformally flat Einstein manifold of positive scalar curvature. Then $M$ is isometric to a 4-dimensional sphere or a complex projective space with the respective standard metric.

Since a 4-dimensional almost Hermitian manifold of pointwise constant holomorphic sectional curvature is self-dual, then by Theorem 4.1 , the manifold $M=(M, J, g)$ under consideration satisfies the conditions of Theorem B. Then from Theorem B we get $T^{*}=0$. On the other hand, we have (see (3.13) of [5])

$$
\int_{M} J^{i a} J^{j b} \nabla_{a} \omega_{b} \nabla_{i} \omega_{j} d M=\int_{M} J^{i a} J^{j b} \nabla_{a} \omega_{b} \nabla_{j} \omega_{i} d M
$$

By (2.2) and (4.7) we obtain

$$
\int_{M}\left\|T^{*}\right\|^{2} d M=4 \int_{M}\|d \omega\|^{2} d M
$$

Hence we have $d \omega=0$, that is $M$ is a locally conformal Kähler surface. Furthermore by (2.2) and (2.3) we have $S=0$, since $M$ is assumed to be Einstein.

This completes the proof of Proposition 2.1 of [3]. 


\section{REFERENCES}

1. N. Hitchin, Kählerian twistor spaces, Proc. London Math. Soc. 43 (1981), 133-150.

2. T. Koda, Self-dual and anti-self-dual Hermitian surfaces, Kodai Math. J. 10 (1987), 335-342.

3. T. Koda and K. Sekigawa, Self-dual Einstein Hermitian surfaces, in Progress in Differential Geometry, Advanced Studies in Pure Mathematics 22 (1993), 123-131.

4. Y. Miyaoka, On the Chern numbers of surfaces of general type, Invent. Math. 42 (1977), 225-237.

5. T. Sato and K. Sekigawa, Hermitian surfaces of constant holomorphic sectional curvature, Math. Z. 205 (1990), 659-668.

6. T. Sato and K. Sekigawa, Hermitian surfaces of constant holomorphic sectional curvature II, Tamkang J. Math. 23 (1992), 137-143.

7. K. Sekigawa, On some 4-dimensional compact almost Hermitian manifolds, J. Ramanujan Math. Soc. 2 (1987), 101-116.

8. F. Tricceri and I. Vaisman, On some 2-dimensional Hermitian manifolds, Math. Z. 192 (1986), 205-216.

Kouei Sekigawa

Department of Mathematics

FACULTY OF SCIENCE

Nigata UnIVERsity

Nigata 950-21

JAPAN
Takashi Koda

Department of Mathematics

FACULTY OF SCIENCE

TOYAMA UNIVERSITY

GoFUKU

TOYAMA 930

JAPAN

E-mail address: koda@sci.toyama-u.ac.jp (Takashi Koda) 